Published in final edited form as:

Leukemia. 2016 December ; 30(12): 2405-2409. doi:10.1038/leu.2016.228.

\title{
Molecular predictors of response in patients with myeloid neoplasms treated with lenalidomide
}

\author{
Eiju Negoro', Tomas Radivoyevitch ${ }^{2}$, Chantana Polprasert ${ }^{1}$, Vera Adema ${ }^{3}$, Naoko Hosono ${ }^{1}$, \\ Hideki Makishima ${ }^{1,4}$, Bartlomiej Przychodzen ${ }^{1}$, Cassandra Hirsch ${ }^{1}$, Michael J. Clemente $^{1}$, \\ Aziz Nazha ${ }^{1}$, Valeria Santini ${ }^{5}$, Kathy L. McGraw ${ }^{6}$, Alan F. List ${ }^{6}$, Francesc Sole ${ }^{3}$, Mikkael A. \\ Sekeres $^{1}$, and Jaroslaw P. Maciejewski ${ }^{1}$ \\ ${ }^{1}$ Department of Translational Hematology and Oncology Research, Taussig Cancer Institute, \\ Cleveland Clinic, Cleveland, $\mathrm{OH}$ \\ ${ }^{2}$ Quantitative Health Sciences, Cleveland Clinic, Cleveland, $\mathrm{OH}$ \\ ${ }^{3}$ Josep Carreras Leukaemia Research Institute, Spain \\ ${ }^{4}$ Department of Pathology and Tumor Biology, Graduate School of Medicine, Kyoto University, \\ Japan \\ ${ }^{5}$ Hematology Unit, AOU Careggi, University of Florence, Italy \\ ${ }^{6}$ Department of Malignant Hematology, H. Lee Moffitt Cancer Center, Tampa, FL
}

\begin{abstract}
Lenalidomide (LEN) treatment leads to red blood cell transfusion independence in approximately two-thirds of anemic myelodysplastic syndrome (MDS) patients with del(5q). (ref. 1) Some patients also experience cytogenetic remissions while in others the del5q abnormality persists.(ref. 1) Furthermore, responses also occur in 25\% of MDS patients without del(5q).(ref. 2) This suggests that in addition to del(5q) other molecular lesions may also be associated with sensitivity to LEN. To date, apart from del $(5 q)$, no reliable predictive factors for LEN responsiveness have been reported, but clinically patients with early MDS, normal/low-risk cytogenetics, or anemia with preserved platelet and neutrophil counts, appear to be better LEN candidates than those with more advanced disease, complex cytogenetics and cytopenias.(ref. 3) On a molecular level, while initial attempts to prospectively identify responders based on gene expression patterns suggested responsespecific signatures,(ref. 4) they have not been translated into an actionable test, and have not been validated in non-del(5q) cases.(ref. 5) Recently, the mechanism of action of LEN has been attributed to induction of ubiquitination and enhanced degradation of casein kinase 1A1 (CK1a) by the E3 ubiquitin ligase cereblon.(ref. 6)
\end{abstract}

\footnotetext{
Users may view, print, copy, and download text and data-mine the content in such documents, for the purposes of academic research, subject always to the full Conditions of use:http://www.nature.com/authors/editorial_policies/license.html\#terms

Correspondence: Jaroslaw P. Maciejewski, M.D., Ph.D., Address: 9500 Euclid Avenue/ R40, Cleveland, Ohio 44195, maciejj@ccf.org, Phone: 2164455962, Fax: 2166362498.

Conflict of Interest

MAS serves on an advisory board for Celgene, Corp. JPM has received speaking honoraria from Celgene.

Supplementary information

Available at Leukemia's website.
} 
To identify new biomarkers of sensitivity to LEN, using next generation sequencing, we determined whether recurrent mutations can help to predict response or resistance to LEN in patients with myeloid malignancies.

We have collected a unique cohort of patients with and without del(5) who underwent routine therapy with LEN and have adequate clinical and molecular annotation. Informed consent in accordance with Institutional Review Board-approved protocols of participating institutions was obtained for sample collection from 137 patients (Supplementary Tables 1, 2), of whom 108 patients were treated with LEN alone (Table 1) and 29 patients received LEN in combination with another agent. WHO classification (2008), IPSS-R, and International Working Group Criteria (2006) were used to define clinical characters. Patients treated at least 2 cycles of LEN were analyzed. Technical details regarding single nucleotide polymorphism array have been previously described.(ref. 7) Multi-amplicon deep sequencing (TruSeq; Illumina, San Diego, CA, USA) was performed for 45 genes most commonly mutated in MDS (Supplementary Table 3). Algorithms for identification of somatic lesions, exclusions of germ line alterations, and removal of technical artifacts were previously reported.(ref. 8) Logistic regression, Fisher's exact test and the Mann-Whitney U test were used to identify covariates that correlated significantly (two-sided a of 0.05 ) with response. Multivariate logistic regression models were identified using backward stepwise selection.

We identified 108 cases of myeloid neoplasms [MDS, MDS/ myeloproliferative neoplasms (MPN), or MPN] treated with LEN alone. The median duration of treatment was 5 months (range 2-66). Patients were sub-grouped into LEN-responder ( $n=55$ ) and LEN-refractory $(n=53)$ cases (Table 1). Initially, we combined isolated del(5q) $(n=29)$, del(5q) with additional cytogenetic abnormalities $(n=17)$, and non del(5q) ( $n=62)$ cases. Patient IPSS-R risks were grouped as either very low/low/intermediate $(92 \%)$ or high/very high $(8 \%)$. Hematologic improvement (HI), partial response (PR), complete response (CR, marrow $\mathrm{CR}$ ), or any response (unknown detail) was achieved in 12 (22\%), 5 (9\%), $19(34.5 \%)$, and $19(34.5 \%)$ of the cases, respectively. In those treated with LEN alone, the del(5q) group overall response rate (ORR) was $65 \%$ (30 responded), while in non-del(5q) patients it was $40 \%$ ( 23 responded) (Supplementary Table $4 \& 5$ ). The relatively favorable response rate in the non-del $(5 q)$ cohort treated in these series may reflect the rational selection of likely responders based on empiric criteria, though this ORR was similar to that of previous studies.(ref. 1,2) Analyses of clinical parameters, including age, absolute neutrophil counts, blast counts, hemoglobin and IPSS-R scores indicated that responders had significantly higher platelets, were more likely to be female, were treated for a longer period of time, had lower IPSS-R scores, and were less likely to have excess blasts (Table 1). The resultant multivariate model of ORR included as covariates platelets and the presence of isolated del(5q); using only the del(5q) cohort the covariates were MDS type and the positivity for PRC2 complex lesions. Within isolated low risk MDS cohort, del(5q) remained the only predictive covariate (Supplementary Table 6). When stratifying based on del(5q) status, in the del(5q) cohort, responders were less likely to have RAEB, and more likely to have lower IPSS-R scores, higher platelets, and a longer treatment period (Supplementary Table 4); in the non-del $(5 \mathrm{q})$ cohort, higher platelets and longer treatment duration also predicted response (Supplementary Table 5). When MDS patients were sub-grouped according to the 
status of disease (low risk: MDS with IPSS-R very low/low/intermediate, MDS/MPN-u, and CMML-1 vs. high risk: MDS with IPSS-R (high/very high), all responses occurred in low risk category, responders likely to have del (5q), isolated del(5q), 5q-MDS, higher platelets and longer treatment duration, while non-responders have normal karyotype and CMML-1 (Supplementary Table $7 \&$ Figure 1). Within cohorts defined by morphologic features such as the presence of ring sideroblasts, uni- $v s$. multi-lineage dysplasia, the responders were not enriched nor a mutational pattern emerged that would predict response (data not shown).

We next examined genomic differences between LEN responders and refractory patients. Cytogenetics, namely complex aberrations, $-7 / \operatorname{del}(7 q),+8,-20 / \operatorname{del}(20 q)$, and $-Y$, were not associated with response to LEN but, as expected, del(5q) and isolated del(5q) was significantly associated with response: odds ratios (OR) of response in all patients were 2.1 $(1,4.7) P=.04$ and $4.2(1.5,13.6) P=.003$ for del5q and isolated del5q, respectively (Figure $1 \mathrm{~A})$ and $2.7(1.2,6.7) P=.02$ and $5.5(2,19) P<.001$ when focused monotherapy (Figure 1B $\&$ Supplementary Figure 2). Both del(5q) long (includes the commonly retained region, CRR) and short (excludes CRR) showed equal sensitivity to LEN (Figure 1A, 1B).

We also applied targeted sequencing (Supplementary Tables 3) or (in selected cases) whole exome sequencing to identify mutations (Supplementary Table 8) that constitute LEN resistance factors. Overall, focusing on OR, the number of mutational events per patient was not associated with responses (responder $v s$. non-responder; OR $1.8 v s .2 .3, P=.35$ ).

Subsequently, we analyzed types of alterations in all cases treated with LEN alone, or with combination therapy. $U 2 A F 1$ ( $\mathrm{OR}<0.5$ ) was overrepresented in refractory cases, while rates for DEAD box RNA helicase combined ( $D D X 41, D D X 54$, and $D H X 29)$, OR $=3.4(1,15.1)$ were significantly higher in responders (Figure 1A). Since the total cohort included patients with combination therapies, to avoid confounding factors, we focused our analyses on cases treated with LEN alone. U2AF1 mutations were significantly associated with failure to respond to LEN ( $0 \%$ vs. $11 \%, P=.01$; Figure 1B). When reverse analysis was performed for all the genes tested for mutations and deletions, $U 2 A F 1$ mutations were also less common among responders ( $0 \%$ response among $U 2 A F 1^{M T} V S .54 \%$ among $U 2 A F 1^{\mathrm{WT}}$ cases; OR < 0.8; Supplementary Figure 2) and 5/6 cases were found in non del(5q) cohort ( $0 \%$ response among $U 2 A F 1^{M T}$ vs. $44 \%$ among $U 2 A F 1^{\mathrm{WT}}$ cases; OR < 1.5; Figure 1C). Previously, MDS patients with $U 2 A F 1$ mutations have been associated with a higher progression rate and poor prognosis. (ref. 9) Similarly, TP53 mutations predict response failures suggesting that del(5q) MDS with $T P 53^{M T}$ might be a clinically important molecular configuration.(ref. 10) However, 2 cases with TP53 mutations were found in the context of del(5q) with complex karyotype (Figure 1B, C) and both, who received LEN alone, were refractory. In contrast, 3/4 (75\%) TP53 mutant or deletion cases who received LEN+Azacitidine combination showed clinical improvement (Figure 1A). When we explored additional LEN sensitivity factors in our del $(5 \mathrm{q})$ cohort, no individual significant somatic mutations were found (Figure 1C), though PRC2 complex genes (EZH2, SUZ12, and DNMT3A) were significantly associated with poor risk by multivariate analysis, and were consistent with the known unfavorable prognosis of $E Z H 2$ mutations.(ref. 11) $E Z H 2$ and $S U Z 12$ are components of the PRC2/EED-EZH2 complex; mutations reduce PRC2 histone methyltransferase activity.(ref. 12) Previously, CSNK1A1 mutation has been shown to convey a poor prognosis in patients with the del(5q) abnormality,(ref. 13) but in our cohort, with a limited number of mutant 
cases, no impact on response to LEN was noted (OR < 34). Recently, $D D X 41$ mutations have been reported to be associated with LEN sensitivity.(ref. 14) Among 7 patients with heterozygous $D D X 41$ mutations or deletions who received LEN alone, the response rate was $57 \%$, OR $=1.3(0.2,9.4)$ (Supplementary Figure 2) and all DDX41 mutants without deletions $(n=3)$ were responders (Figure $1 \mathrm{~B})$. In the non-del $(5 \mathrm{q})$ cohort, DEAD box RNA helicase ( $D D X 41, D D X 54$, and $D H X 29)$ mutations/deletions were significantly more common in responders $(P=.02)$.

Grouping clone sizes to identify dominant likely ancestral events (SF3B1, TET2, JAK2, GPR98, PRPF8, STAG2, DNMT3A, and ZRSR2) did not yield significant results (Supplementary Table $9 \&$ Figure 3). In a recent study of patients treated with LEN and erythropoietin, combined predictors that do not include mutation status were described, and analysis of clonal architecture showed effects on subclonal distribution, whereas loss of responsiveness was associated with the re-expansion of the dominant subclone.(ref. 15)

In conclusion, in addition to the presence of $\operatorname{del}(5 q)$, gender, platelet counts, treatment period, MDS type, and IPSS-R, the U2AF1 mutation in the entire cohort, mutations in TP53 in the del(5q) cohort, and DEAD box RNA helicases in the non-del(5q) cohort grouped with responses to LEN.

\section{Supplementary Material}

Refer to Web version on PubMed Central for supplementary material.

\section{Acknowledgments}

This work was supported in part by National Institutes of Health grants R01HL118281 and Celgene Award RV-TRMYELOID-PI-004593 to J.P. Maciejewski and by the Edward P. Evans Foundation to JPM and MAS. Financial support. This work was supported in part by a grant from the Instituto de Salud Carlos III, Ministerio de Economia y Competividad, Spain (PI 11/02010 and PI/14/00013); by the Red Temática de Investigación Cooperativa en Cáncer (RTICC, FEDER) (RD12/0036/0044); Sociedad Española Hematología y Hemoterapia; 2014 SGR225 (GRE) Generalitat de Catalunya, by José Carreras Leukämie-Stiftung, Ref. AR 14/34; economical support from Fundació Internacional Josep Carreras and from Celgene Spain. The research leading to this invention has received funding from "la Caixa" Foundation.

\section{References}

1. List A, Dewald G, Bennett J, Giagounidis A, Raza A, Feldman E, et al. Lenalidomide in the myelodysplastic syndrome with chromosome 5q deletion. N Engl J Med. 2006 Oct 5.355:14561465. [PubMed: 17021321]

2. Raza A, Reeves JA, Feldman EJ, Dewald GW, Bennett JM, Deeg HJ, et al. Phase 2 study of lenalidomide in transfusion-dependent, low-risk, and intermediate-1 risk myelodysplastic syndromes with karyotypes other than deletion 5q. Blood. 2008 Jan 1.111:86-93. [PubMed: 17893227]

3. Sekeres MA, Maciejewski JP, Giagounidis AA, Wride K, Knight R, Raza A, et al. Relationship of treatment-related cytopenias and response to lenalidomide in patients with lower-risk myelodysplastic syndromes. J Clin Oncol. 2008 Dec 20.26:5943-5949. [PubMed: 19018091]

4. Ebert BL, Galili N, Tamayo P, Bosco J, Mak R, Pretz J, et al. An erythroid differentiation signature predicts response to lenalidomide in myelodysplastic syndrome. PLoS Med. 2008 Feb.5:e35. [PubMed: 18271621]

5. Santini V, Almeida A, Giagounidis A, Gropper S, Jonasova A, Vey N, et al. Randomized Phase III Study of Lenalidomide Versus Placebo in RBC Transfusion-Dependent Patients With Lower-Risk 
Non-del(5q) Myelodysplastic Syndromes and Ineligible for or Refractory to ErythropoiesisStimulating Agents. J Clin Oncol. 2016 Sep 1.34:2988-2996. [PubMed: 27354480]

6. Sardnal V, Rouquette A, Kaltenbach S, Bally C, Chesnais V, Leschi C, et al. A G polymorphism in the CRBN gene acts as a biomarker of response to treatment with lenalidomide in low/int-1 risk MDS without del(5q). Leukemia. 2013 Jul.27:1610-1613. [PubMed: 23434730]

7. Maciejewski JP, Tiu RV, O'Keefe C. Application of array-based whole genome scanning technologies as a cytogenetic tool in haematological malignancies. Br J Haematol. 2009 Sep. 146:479-488. [PubMed: 19563474]

8. Makishima H, Yoshida K, Nguyen N, Przychodzen B, Sanada M, Okuno Y, et al. Somatic SETBP1 mutations in myeloid malignancies. Nat Genet. 2013 Aug.45:942-946. [PubMed: 23832012]

9. Yoshida K, Sanada M, Shiraishi Y, Nowak D, Nagata Y, Yamamoto R, et al. Frequent pathway mutations of splicing machinery in myelodysplasia. Nature. 2011 Oct 6.478:64-69. [PubMed: 21909114]

10. Jadersten M, Saft L, Smith A, Kulasekararaj A, Pomplun S, Gohring G, et al. TP53 mutations in low-risk myelodysplastic syndromes with del(5q) predict disease progression. J Clin Oncol. 2011 May 20.29:1971-1979. [PubMed: 21519010]

11. Hosono N, Makishima H, Jerez A, Yoshida K, Przychodzen B, McMahon S, et al. Recurrent genetic defects on chromosome 7q in myeloid neoplasms. Leukemia. 2014 Jun.28:1348-1351. [PubMed: 24429498]

12. Score J, Hidalgo-Curtis C, Jones AV, Winkelmann N, Skinner A, Ward D, et al. Inactivation of polycomb repressive complex 2 components in myeloproliferative and myelodysplastic/ myeloproliferative neoplasms. Blood. 2012 Feb 2.119:1208-1213. [PubMed: 22053108]

13. Schneider RK, Adema V, Heckl D, Jaras M, Mallo M, Lord AM, et al. Role of casein kinase 1A1 in the biology and targeted therapy of del(5q) MDS. Cancer Cell. 2014 Oct 13.26:509-520. [PubMed: 25242043]

14. Polprasert C, Schulze I, Sekeres MA, Makishima H, Przychodzen B, Hosono N, et al. Inherited and Somatic Defects in DDX41 in Myeloid Neoplasms. Cancer Cell. 2015 May 11.27:658-670. [PubMed: 25920683]

15. Chesnais V, Renneville A, Toma A, Lambert J, Passet M, Dumont F, et al. Effect of lenalidomide treatment on clonal architecture of myelodysplastic syndromes without 5q deletion. Blood. 2016 Feb 11.127:749-760. [PubMed: 26626993] 

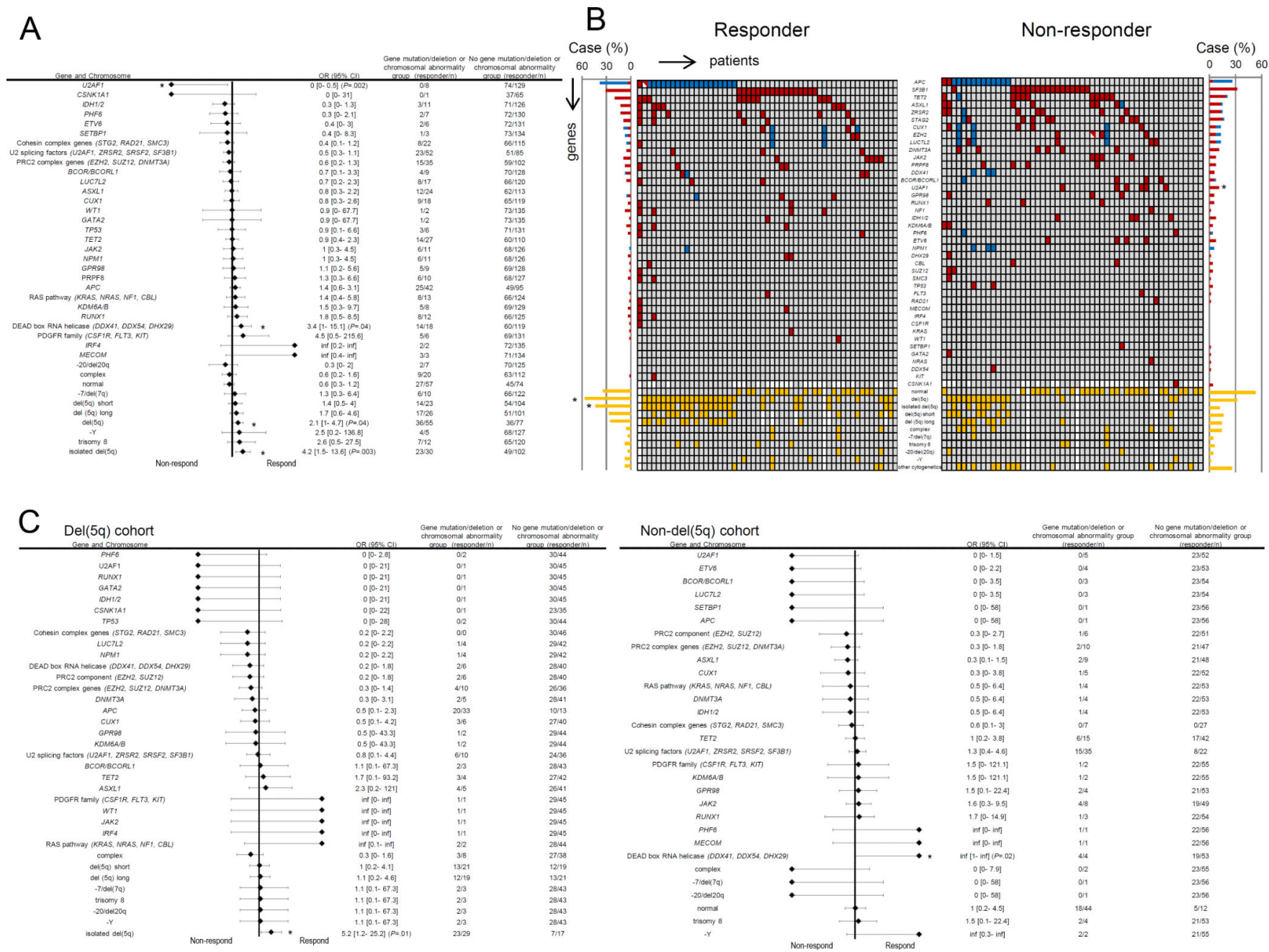

Figure 1.

Correlations of gene mutation/deletion and cytogenetics abnormality with Lenalidomide response. (A) Response rate to LEN alone and LEN combination therapy in patients with gene mutations/deletions or cytogenetic abnormalities compared with wild type cases. (B) Spectrum of mutations and deletions in myeloid malignancy between responder $(\mathrm{n}=55)$ and non-responder $(\mathrm{n}=53)$ to Lenalidomide (LEN) monotherpay, * mutation or cytogenetic abnormality; $\mathrm{p}<.05$. Red, blue, yellow, gray and white indicate mutations, deletions, cytogenetic abnormalities, wild type and no data respectively. Triangle indicated mutation + deletion of the corresponding genes affected by hypomorhic/loss of function mutations. (C) Response rate to LEN monotherapy in $\operatorname{del}(5 q)$ and non-del $(5 q)$ patients with gene mutations/ deletions or cytogenetic abnormalities compared with wild type cases; $*<.05$. del(5q) short: excluding commonly retained regions (CRR; q11.1-q14.2 and/or q34-qter), del(5q) long: including CRR. Inf: infinity. 


\section{Table 1}

Clinical Characteristics of patients of Myeloid malignancy treated with LEN monotherapy

\begin{tabular}{|c|c|c|c|}
\hline & Responder & Non-responder & $P$ \\
\hline Sample size for sequencing & 55 & 53 & \\
\hline \multicolumn{4}{|l|}{ Diagnosis } \\
\hline $\operatorname{MDS}(n=91)$ & 48 & 43 & .2 \\
\hline Low risk (RA, RARS, RCMD, 5q-MDS) & 42 & 29 & $.009^{*}$ \\
\hline RA (FAB criteria) & 8 & 5 & .55 \\
\hline RARS & 6 & 7 & .78 \\
\hline RCMD & 9 & 14 & .24 \\
\hline 5q-MDS & 19 & 3 & $<.001^{*}$ \\
\hline High risk (RAEB-1) & 2 & 11 & $.008^{*}$ \\
\hline MDS/MPN $(n=10)$ & 3 & 7 & .2 \\
\hline CMML-1 & 0 & 4 & .06 \\
\hline MDS/MPN unclassifiable & 3 & 3 & 1 \\
\hline $\operatorname{MPN}(n=7)$ & 4 & 3 & 1 \\
\hline Myelofibrosis & 4 & 3 & 1 \\
\hline \multicolumn{4}{|l|}{ Demographics } \\
\hline \multicolumn{4}{|l|}{ Age $(n=40$ vs. $n=46)$} \\
\hline Median (range) & $69(20-90)$ years & $72(30-85)$ years & .95 \\
\hline \multicolumn{4}{|l|}{ Gender ( $n=41$ vs. $n=46)$} \\
\hline Female & 25 & 17 & $.03^{*}$ \\
\hline \multicolumn{4}{|l|}{ IPSS-R ( $n=48$ vs. $n=42)$} \\
\hline High risk (very high, high) & 0 & 7 & $.008^{*}$ \\
\hline Low risk (intermediate, low, very low) & 48 & 35 & $.008^{*}$ \\
\hline \multicolumn{4}{|l|}{ Blood counts before treatment ( $n=36$ vs. $n=38$ ) } \\
\hline Hemoglobin level, median (quartile 1-3) & $8.6(8-9.6) \mathrm{g} / \mathrm{dl}$ & $8.9(8.0-10.1) \mathrm{g} / \mathrm{dl}$ & .56 \\
\hline Neutrophil count, median (quartile 1-3) & $1.8(1.1-3.0) \times 10^{9} / 1$ & $1.8(1.2-4.0) \times 10^{9} / 1$ & .39 \\
\hline Platelet count, median (quartile 1-3) & $284(140-366) \times 10^{9} / 1$ & $85(33-196) \times 10^{9} / 1$ & $<.001^{*}$ \\
\hline \multicolumn{4}{|l|}{ Bone marrow ( $n=34$ vs. $n=37$ ) } \\
\hline Blast $>5 \%$ & 2 & 6 & .15 \\
\hline \multicolumn{4}{|l|}{ Cyotogenetics ( $n=53$ vs. $n=50)$} \\
\hline Normal & 18 & 26 & .07 \\
\hline Any abnormal cytogenetics & 35 & 24 & .07 \\
\hline $\operatorname{Del}(5 q)$ with or without other mutations & 30 & 16 & $.02 *$ \\
\hline Isolated del(5q) & 23 & 6 & $<.001 *$ \\
\hline $\operatorname{Del}(5 q)$ short & 13 & 8 & .2 \\
\hline $\operatorname{Del}(5 q)$ long & 12 & 7 & .2 \\
\hline
\end{tabular}




\begin{tabular}{lcccc}
\hline & Responder & Non-responder & $P$ \\
\hline \multicolumn{1}{c}{ Complex } & 3 & 7 & .19 \\
\hline -7/del(7q) with or without other mutations & 2 & 2 & 1 \\
\hline Trisomy 8 with or without other mutations & 4 & 3 & 1 \\
\hline -20/del(20q) with or without other mutations & 2 & 2 & 1 \\
\hline \multicolumn{1}{r}{$Y$ with or without other mutations } & 4 & 1 & .36 \\
\hline Other & 4 & 13 & N/A \\
\hline Treatment period $(n=30$ vs. $n=36)$ & & & & \\
\hline Median (range) & $12.5(2-66)$ months & $3.5(2-19)$ months & $<.001$ \\
\hline Response & & N/A & N/A \\
\hline Complete response (CR/ marrow CR) & 12 & N/A & N/A \\
\hline Partial response (PR) & 5 & N/A & N/A \\
\hline Hematologic improvement (HI) & 19 & N/A & N/A \\
\hline Unknown detail & 19 & &
\end{tabular}

IPSS-R: Revised International Prognostic Scoring System, LEN: Lenalidomide, Aza: Azacytidine MDS: Myelodysplastic syndrome, MPN: Myeloproloferative neoplasms, RA: Refractory anemia RCUD: Refractory cytopenias with unilineage dysplasia, RARS: Refractory anemia with ring sideroblasts RCMD: Refractory cytopenia with multilineage dysplasia, RAEB: Refractory anemia with excess blasts CMML: Chronic myelomonocytic leukemia, MDS type is unknowen in 7 cases. del(5q) short: excluding commonly retained regions (CRR; q11.1-q14.2 and/or q34-qter), del(5q) long: including CRR, N/A; Not applicable,

${ }^{*}<<.05$ 